\title{
Study of serum and synovial fluid Dickkopf- 1 levels in patients with primary osteoarthritis of the knee joint in correlation with disease activity and severity
}

Noha Hosni Ibrahim*, Samia Mohammed Abdel-Monem, Abdel-Wahab S. E. Elbarashy, Heba A. W. Elhussieny and Rasha A. H. Elsayed

\begin{abstract}
Background: Osteoarthritis $(\mathrm{OA})$ is a typical complex degenerative articular ailment that shows focal cartilage loss, new bone formation with involvement of entire joint tissues. Dkk-1 assumes a job in controlling the pattern of bone repair and regeneration in both OA and RA. This study aimed to determine serum and synovial fluid levels of Dickkopf-1 (Dkk-1) in patients with primary OA of the knee joint and study their correlations with disease activity and severity. This study included 45 patients, 30 of them were diagnosed as primary knee OA. Fifteen rheumatoid arthritis patients as well as 15 healthy subjects were enrolled in the study as control groups, serum and synovial levels of Dkk-1 were estimated utilizing the ELISA technique.
\end{abstract}

Results: Serum levels of Dkk-1 were significantly higher in OA patients than healthy subjects $(p<0.001)$, although it was even significantly higher in RA patients than OA patients $(p<0.001)$. There was a highly significant decrease in the median synovial level of Dkk-1 in OA patients compared to the RA control group $(p<0.001)$. There was a highly statistically significant inverse correlation between circulating as well as synovial fluid Dkk-1 levels and radiological disease grading in knee OA $(p<0.001)$. There was a statistically significant decrease in serum levels of Dkk-1 in patients with severe OA (grade 3, 4) compared to those with mild OA (Grade 2) $(p<0.001)$.

Conclusion: Dkk-1 is an interesting marker that is related to articular disease .It could play an important role in decelerating the degenerative process of $\mathrm{OA}$ and can reflects radiographic severity of the disease as well.

Keywords: Primary osteoarthritis, Dickkopf-1, Dkk-1, WOMAC index

\section{Background}

Osteoarthritis (OA) is a degenerative joint disorder prompting articular cartilage degradation, subchondral loss, and bone remodeling leading to joint space narrowing, pain, and loss of function [1]. OA is the most widely recognized disease of knee particularly in the middle to old ages [2]. Globally, it affects about $3.3 \%$ to $3.6 \%$ of the population resulting in moderate to severe disability making it one of the most incapacitating illnesses around the globe [3]. Inflammation in OA is chronic and gradually progressive in contrast to inflammatory arthritis, involving mainly innate immune mechanisms. Synovitis is a usual feature in OA that can occur in early stages of the disease but it is increasingly common toward the more advanced stages and severe disease [4].

* Correspondence: drnonon@yahoo.com

Benha Faculty of Medicine, Benha, Egypt

(C) The Author(s). 2020 Open Access This article is licensed under a Creative Commons Attribution 4.0 International License, which permits use, sharing, adaptation, distribution and reproduction in any medium or format, as long as you give appropriate credit to the original author(s) and the source, provide a link to the Creative Commons licence, and indicate if changes were made. The images or other third party material in this article are included in the article's Creative Commons licence, unless indicated otherwise in a credit line to the material. If material is not included in the article's Creative Commons licence and your intended use is not permitted by statutory regulation or exceeds the permitted use, you will need to obtain permission directly from the copyright holder. To view a copy of this licence, visit http://creativecommons.org/licenses/by/4.0/. 
Several cytokines have been researched in OA either circulating or detected in synovial fluid of joints scanning for biochemical markers that could influence disease severity and progression. Several studies on the possible association between serum and synovial fluid levels of Dkk-1 with disease activity in knee OA have been postulated [5].

Dkk-1 and osteoprotegerin (OPG) which represent the key components of bone metabolism in normal joint bone, interact with Wnt signalling pathway, balancing between bone absorption and bone formation [6]. Families of both activators and inhibitors regulate the Wnt signalling pathway. The Dkk proteins are considered one of inhibitors family, Dkk-1 plays a role in controlling the pattern of bone repair and regeneration in both $\mathrm{OA}$ and RA [7]. It has been found to delay osteophytes formation and subchondral bone remodeling and is a potent negative modulator of osteoblast differentiation [8].

\section{Aim of the work}

The aim of this work is to determine serum and synovial fluid levels of Dickkopf-1 (Dkk-1) in patients with primary OA of the knee joint and study their correlations with disease activity and severity to explicate their clinical value in the disease.

\section{Methods}

This study is conducted upon 60 individuals used to attend the outpatient's clinic and inpatients' department of Rheumatology, Rehabilitation and Physical Medicine, Benha University Hospitals. These subjects are divided into 3 groups:

Group (I): Thirty patients with primary knee OA, fulfilling the 2016 revised criteria for the diagnosis of knee OA [2].

Two control groups of matching age and sex to group (I):

Group (II): Fifteen patients with RA clinically diagnosed and classified according to the American College of Rheumatology/European league against Rheumatism (ACR/EULAR) classification criteria for RA [9].

Group (III): Fifteen apparently healthy adult.

\section{Criteria for exclusion}

Subjects were excluded from the study if they had apparent causes for secondary OA, e.g., knee joint deformity or morbid obesity, other systemic inflammatory or autoimmune disorders, previous knee injury or joint infection, history of corticosteroid medication $\leq 6$ months.

All patients were subjected to full medical history, clinical examination, functional assessment using the WOMAC index [10] and the KOFUS [11], laboratory investigations including $\mathrm{CBC}$, ESR and CRP. Weight bearing plain $\mathrm{X}$-rays were done for the affected knee to assess radiological severity of the disease using Kellgren and Lawrence grading [12]. Serum and synovial fluid samples were obtained from all OA and RA patients to measure levels of Dkk-1 using the ELISA technique [13].

\section{Ethical considerations}

Written consents according to Helsinki Declaration were obtained from all patients and control subjects before participation in the study that was approved by the ethical committee of Benha Faculty of Medicine.

\section{Statistical methods}

The quantitative variables are presented by mean and standard deviation while qualitative variables are presented by the median, interquartile range, frequencies and percentages, respectively. Data management and statistical analysis were done using the Statistical Package for Social Sciences (SPSS) computer program vs.25. (IBM, Armonk, NY, USA). Suitable analysis was done according to the type of data obtained for each parameter. Comparisons between two groups are done using Mann-Whitney test. Three groups' comparisons are done using Kruskal Wallis test for numerical data. Categorical data is compared using chi-square test or Fisher's exact test when appropriate. Pairwise analysis is done in case of significant overall effect and is adjusted for multiple comparisons. Correlation analysis is done using Pearson's or Spearman's correlation, " $r$ " is the correlation coefficient. It ranges from -1 to +1 . Minus one indicates perfect negative correlation. Plus, one indicates perfect positive correlation while 0 indicates no correlation. All $p$ values are two sided. $p$ values equal or less than 0.05 are considered significant.

\section{Results}

This study included 30 primary knee OA patients [26 females (86.6\%) and 4 males (14.4\%)] (group I) diagnosed according to the 2016 revised criteria, for the diagnosis of knee OA as well as 30 controls. The controls were divided into 2 groups; 15 RA female patients (group II) and 15 apparently healthy adult females (group III).

The mean age of OA patients was $51.4 \pm 5.8$ years, their mean disease duration was $8.1 \pm 4$.4 years and their mean BMI was $32.1 \pm 3.8 \mathrm{~kg} / \mathrm{m}^{2}$. RA controls had a mean age of $48.9 \pm 11.7$ years, while healthy controls had a mean age of $48.2 \pm 5.2$ years (Table 1 ).

Clinically, 9 OA patients (30\%) had unilateral knee affection, while 21 patients $(70 \%)$ had bilateral affection. Twenty-five patient (83.3\%) had morning stiffness and 11 patients (36.7\%) presented with knee effusion. The mean WOMAC index was $54.6 \pm 18.0$, while the KOFUS was $6.8 \pm 4.6$. Kellgren and Lawrence X-ray grading score of group I revealed 2 patients $(6.7 \%)$ having grade II, 17 patients $(56.7 \%)$ with grade III $(56.7 \%)$ and 11 patients with grade IV (36.7\%). Group II showing 3 patients with grade (20.0\%), 9 patients with grade III (60.0\%), and 3 patients 
Table 1 Demographic data of the studied groups; showing no statistically significant difference regarding age, gender, BMI between the 3 groups and also insignificant statistical difference in the disease duration between the 2 groups

\begin{tabular}{|c|c|c|c|c|c|}
\hline & & Group I (30) & Group II (15) & Group III (15) & $p$ value \\
\hline \multirow[t]{2}{*}{ Age (years) } & Range & $42-64$ & $28-74$ & $39-56$ & 0.34 \\
\hline & Mean \pm SD & $51.4 \pm 5.8$ & $48.9 \pm 11.7$ & $48.2 \pm 5.2$ & \\
\hline \multirow[t]{2}{*}{ Gender } & Males, $n(\%)$ & $4(13.3)$ & $0(0.0)$ & $0(0.0)$ & 0.17 \\
\hline & Females, $n(\%)$ & $26(86.7)$ & $15(100.0)$ & $15(100.0)$ & \\
\hline \multirow[t]{2}{*}{$\mathrm{BMI}\left(\mathrm{kg} / \mathrm{m}^{2}\right)$} & Range & $25-39.9$ & $27.6-33.2$ & $27-33.8$ & 0.094 \\
\hline & Mean \pm SD & $32.1 \pm 3.8$ & $30.7 \pm 2.3$ & $30.1 \pm 1.6$ & \\
\hline \multirow[t]{2}{*}{ Disease duration } & Range & $2-16$ & $2-14$ & - & 0.17 \\
\hline & Mean \pm SD & $8.1 \pm 4.4$ & $6.3 \pm 3.6$ & - & \\
\hline
\end{tabular}

Kruskal-Wallis test was used for age, BMI and disease duration. Gender was compared using Fisher's exact test

with grade IV (20.0\%). In Table 2, serum and synovial Dkk-1 level showed an overall statistically significant difference between the study groups $\left(p^{<} 0.001\right)$. It reveals that group II has the highest serum level among the 3 studied groups and that it also has a higher synovial level than group I.

\section{Correlative analysis}

Regarding correlations of median serum Dkk-1 levels with mean demographic, clinical and laboratory parameters of OA group are presented in Table 3.

There was significant negative correlations of median serum Dkk-1 level with joint stiffness, physical function as well as total WOMAC index $(r=-0.37,-0.41$, and - 0.37, respectively, $p=0.047,0.023$, and 0.047 respectively).

There was a highly significant positive correlation between median serum Dkk-1 and median synovial Dkk-1 $(r=0.62, p<0.001)$.

There were non-significant correlations of mean serum Dkk-1 level with other study parameters (age, BMI, disease duration, pain, ESR, and KOFUS score).

Correlations of median synovial Dkk-1 level with mean demographic, clinical and laboratory parameters in OA group are displayed in Table 4.

There were highly statistically significant negative correlations of median synovial Dkk-1 level with mean disease duration $(r=-0.40, p=-0.029)$, mean WOMAC

Table 2 Serum and synovial levels of Dkk-1 in the studied groups showing that group II has the highest serum level among the 3 studied groups and that it also has a higher synovial level than group I

\begin{tabular}{lllll}
\hline Variables & $\begin{array}{l}\text { Group I } \\
(n=30)\end{array}$ & $\begin{array}{l}\text { Group II } \\
(n=15\end{array}$ & $\begin{array}{l}\text { Group III } \\
(n=15)\end{array}$ & $p$ value \\
\hline Serum Dkk-1 (ng/ml) & 4.12 & 8.39 & 1.9 & $<0.001^{* *}$ \\
median (IQR) & $(2.86-5.15)$ & $(7.0-18.6)$ & $(1.72-2.1)$ & \\
Synovial Dkk-1 (ng/ml) & 0.447 & 3.7 & - & $<0.001^{* *}$ \\
median (IQR) & $(0.129-1.13)$ & $(3.4-7.0)$ & & \\
\hline
\end{tabular}

IQR inter-quartile range. Kruskal-Wallis test was used for serum DKK-1. MannWhitney $U$ test was used for synovial DKK-1 index $(r=-0.624, p=0.001)$, and mean $\mathrm{X}$-ray grading $(r=-0.599, p<0.001)$ (Figs. 1 and 2).

There were non-significant correlations between median synovial Dkk-1 level and other study parameters (age, BMI, pain, ESR, and KOFUS score) (Table 4).

\section{Discussion}

Complex cellular processes, such as proliferation and differentiation are controlled by Wnt signalling pathway [14]. Dkks which are Wnt modulators are likewise associated with this exceptionally entwined utilitarian system [15]. Dkk-1 is a group of cysteine-rich proteins comprising Dkk-1, Dkk-2, Dkk-3, Dkk-4, and a unique Dkk-3 related protein [16]. Dkk-1 acts as a natural inhibitor of Wnt signalling pathway that assumes significant jobs in

Table 3 Correlations of mean serum Dkk-1 level with mean demographic, clinical, and laboratory parameters in osteoarthritis group; negative correlations were observed between serum Dkk1 and pain score, joint stiffness and physical function, and positive correlation with synovial Dkk-1

\begin{tabular}{lll}
\hline Group I (30) & \multicolumn{2}{l}{ S Dkk-1 (ng/ml) } \\
\cline { 2 - 3 } & Correlation coefficient (rho) & $p$ value \\
\hline Age/years & -0.31 & 0.098 \\
BMI $\left(\mathrm{kg} / \mathrm{m}^{2}\right)$ & -0.12 & 0.52 \\
Disease duration/years & -0.13 & 0.49 \\
ESR (mm/h) & 0.12 & 0.54 \\
Pain & -0.19 & 0.32 \\
Joint stiffness & -0.37 & $0.047^{*}$ \\
Physical function & -0.41 & $0.023^{*}$ \\
Total score of WOMAC index & -0.37 & $0.047^{*}$ \\
KOFUS score & -0.312 & 0.093 \\
X-ray grading & -0.31 & 0.09 \\
Synovial Dkk-1 & 0.62 & $<0.001^{* *}$ \\
\hline
\end{tabular}

rho correlation coefficient, $B M I$ body mass index, ESR erythrocyte sedimentation rate, WOMAC Western Ontario and McMaster Universities Arthritis Index, KOFUS knee osteoarthritis flare up score

*Significant

**Highly significant 
Table 4 Correlations of median synovial Dkk-1 (ng/ml) level with mean demographic, clinical and laboratory parameters in osteoarthritis group (I)

\begin{tabular}{lll}
\hline Group I (30) & \multicolumn{2}{l}{ Synovial Dkk-1 $(\mathrm{ng} / \mathrm{ml})$} \\
\cline { 2 - 3 } & Correlation coefficient $(r h o)$ & $p$ value \\
\hline Age/years & -0.36 & 0.052 \\
BMI $\left(\mathrm{kg} / \mathrm{m}^{2}\right)$ & -0.20 & 0.29 \\
Disease duration/years & -0.40 & $0.029^{*}$ \\
ESR $(\mathrm{mm} / \mathrm{h})$ & -0.02 & 0.91 \\
Pain & -0.32 & 0.085 \\
Joint stiffness & -0.53 & $0.002^{* *}$ \\
Physical function & -0.60 & $<0.001^{* *}$ \\
Total score of WOMAC index & -0.57 & $0.001^{* *}$ \\
KOFUS score & -0.258 & 0.169 \\
X- ray grading & -0.60 & $<0.001^{* *}$
\end{tabular}

rho Spearman's correlation coefficient, $B M I=$ body mass index, ESR erythrocyte sedimentation rate, WOMAC Western Ontario and McMaster Universities Arthritis Index, KOFUS knee osteoarthritis flare up score *Significant

**Highly significant

vertebrate embryogenesis [14]. Circulating Dkk-1 is present in RA, AS, and OA, while studies showed that Dkk-1 was expressed in synovial cells, articular cartilage chondrocytes, and subchondral bone osteoblasts in OA knees [17-20].

In our study, serum and synovial fluid Dkk-1 levels were measured in a well-defined knee OA population, and according to our results, serum levels of Dkk-1 were significantly higher in OA patients than healthy subjects $(p<0.001)$, although it was even significantly higher in RA patients than OA patients $(p<0.001)$. VoorzangerRousselot and Coworkers [21], Honsawek et al. [22] and Min et al. [6] reported that the Dkk-1 levels were significantly lower in their OA patients than that of the healthy controls, while Hassan and Behiry [23], found that there was no statistically significant difference in serum Dkk-1 levels between knee OA patients and healthy controls. This difference may be attributed to ethnic discrepancy, also different disease grading and varied disease presentations. Theologis et al. [13], revealed that synovial Dkk-1 levels were significantly higher in OA, compared to the control group. We did not get synovial fluid from healthy subjects, but regarding RA controls Dkk-1 was significantly higher than OA patients $(p<0.001)$. This is in agreement of the study done by Seror and his colleagues in 2016 [24] which was conducted in a large cohort of early onset RA patients and showed an increase in Dkk-1 serum and synovial levels and hence, it found to be a useful marker for disturbed structural integrity and erosive condition.

Diarra et al. [25], stated that Dkk-1 levels in ankylosing spondylitis, an inflammatory spondyloarthropathy characterized by osteophytes formation, were very low and even below the levels in healthy subjects, suggesting a role of Dkk-1 in differential remodeling of human joint architecture. The study revealed a highly statistically significant inverse correlation between circulating as well as synovial fluid Dkk-1 levels and radiological disease grading in knee OA $(r=-0.599, p<0.001)$ as well as with mean disease duration $(r=-0.40, p=-0.029)$.

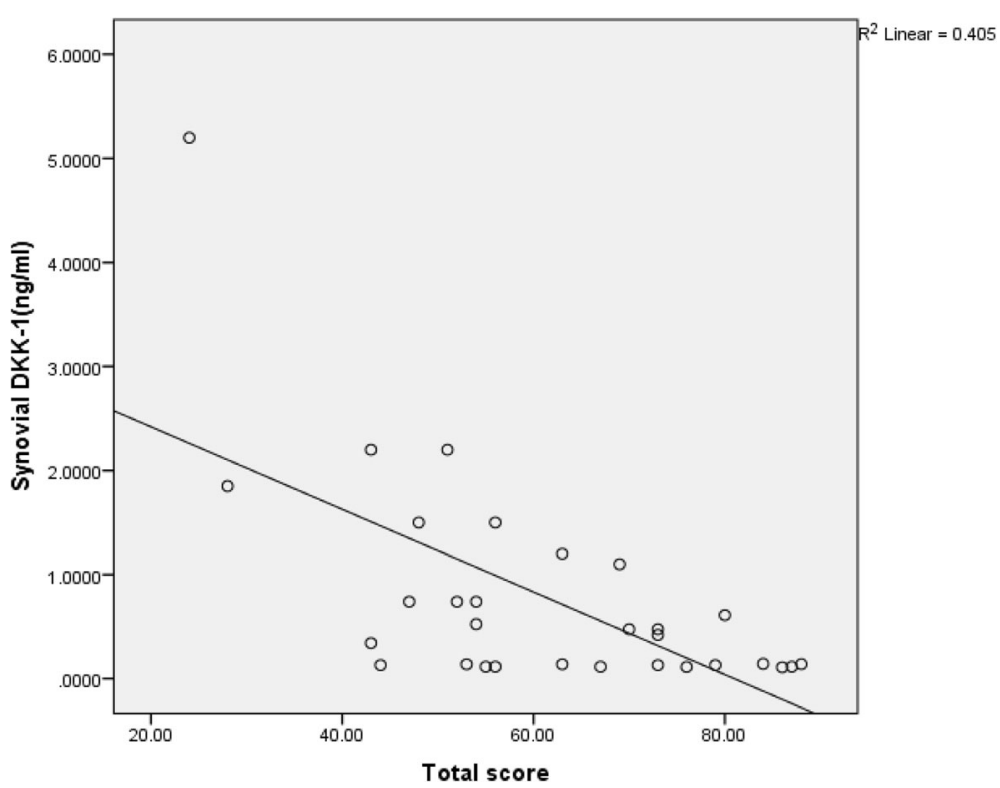

Fig. 1 Correlation between synovial Dkk-1 levels and total WOMAC index score in primary knee OA patients 


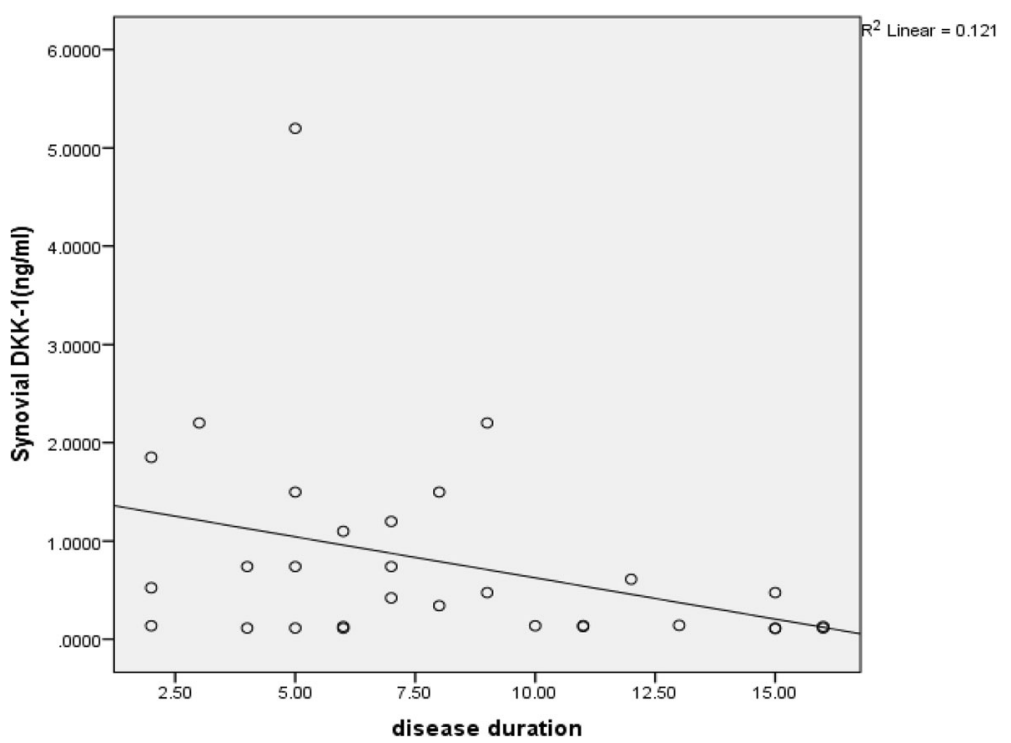

Fig. 2 Correlation between synovial Dkk-1 levels and disease duration in primary knee OA patients

This was approved by Lane et al. [26], who reported that increased circulating levels of Dkk-1 appeared to be associated with delayed radiographic progression of hip OA in elderly women. This is in agreement with our results where we detected a highly statistically significant negative correlation between median synovial Dkk-1 level and mean X-ray grading $(r=-0.599, p<0.001)$, However, Theologis et al. (2017) reported that synovial fluid Dkk-1 levels were significantly higher in advanced knee OA patients compared to early OA patients.

Our study revealed a significantly negative correlation of serum as well as synovial fluid Dkk-1 levels with joint stiffness, physical function as well as total WOMAC index. Honsawek et al. [22], also found an inverse correlation between circulating as well as synovial fluid Dkk-1 levels and disease progression in knee OA. Circulating levels of Dkk-1 were higher in early knee OA patients (KL grade 2) compared to advanced knee OA (KL grade 4). Dkk-1 has been demonstrated to delay osteophytes formation and subchondral bone remodeling and is a potent negative modulator of osteoblast differentiation [8]. So, in mild to moderate knee OA patients with high serum and synovial fluid levels of Dkk-1, Dkk-1 may be able to inhibit bone remodeling around the osteoarthritic joint. Honsawek et al. [22], proposed that Dkk-1 can delay articular cartilage loss. Min et al. [6] revealed that an increase in serum level of Dkk-1 was significantly associated with a reduced risk of suffering OA $(\mathrm{OR}=$ 0.999, $p<0.01$ ).

The role of Dkk-1 in defending cartilage has not been recognized yet, according to Leijten and his colleagues [27], Dkk-1 acts as natural brakes on hypertrophic differentiation in articular cartilage. hypertrophic differentiation of articular cartilage can contribute to the progress of OA. However, our study limitation is the small sample size of $\mathrm{OA}$ and RA patients.

Dkk-1 is an interesting marker that is related to articular disease although it does not correlate with activity or severity of OA, its highly statistically significant inverse correlation between circulating as well as synovial fluid Dkk-1 levels and radiological disease grading in OA and its higher levels in RA patients compared to OA reflects the ongoing response of the body to repair the articular damage that happens in both diseases. The higher level in the moderate OA reflects that this group has enough damage to elicit a compensatory mechanism but not so severe to lead to failure of this reparative pathways. The study recommends further research to see if the Dkk-1 could have a therapeutic implication to support the cartilage in OA as well as in RA

\section{Conclusions}

Dkk-1 is an interesting marker that is related to articular disease. It could play an important role in decelerating the degenerative process of OA and can reflect radiographic severity of the disease as well. The study reflects the effective trials of the homeostatic balance of the arthritic joints to retard the progression of the ongoing joint degeneration. This role of the Dkk-1 suggests that it could have a therapeutic implication for treatment of primary and secondary OA. The study recommends further research to see if the Dkk-1 could have a therapeutic implication to support the cartilage in OA as well as in RA. Future research should be focused on detection of a possible role of Dkk-1 in the pathogenesis of chronic degenerative joint disorder as well as 
inflammatory disorders such as RA. Further studies using ultrasonography of the affected joints determine the correlation of the articular finding such as synovitis, effusion, erosive changes, and osteophyte formation to Dkk-1 levels. Also, future research investigating the targeted inhibition of Wnt signaling, aiming to the development of effective therapeutic approaches to control the progression of OA and RA should be the focus of in vitro as well as in vivo future research work.

\section{Abbreviations}

Dkk-1: Dickkopf-1; KOFUS: Knee osteoarthritis flare up score;

OA: Osteoarthritis; RA: Rheumatoid arthritis; WOMAC: Western Ontario and McMaster Universities Osteoarthritis Index

\section{Acknowledgements}

Not applicable

\section{Authors' contributions}

$\mathrm{NH}$ : idea suggestion, put study design, data collection and statistical analysis and contributed in methodology, results and discussion writing and manuscript revision. SM: contributed in background, objectives, results and discussion writing. AS: contributed in background, objectives results, discussion and conclusion writing. HA: obtaining patients samples and data collection and statistical analysis sharing, also contributed in methodology, results, discussion and conclusion writing. RA: Laboratory work including measurement of Dkk-1 in serum and synovial fluid using ELISA, also contributed in methodology and results writing. All authors read and approved the final manuscript.

\section{Funding}

The study did not receive any public or governmental funding.

\section{Availability of data and materials}

The datasets used and/or analysed during the current study are available from the corresponding author on reasonable request.

\section{Ethics approval and consent to participate}

Done, the committee's reference number : 1042018, date: 10-4-2018 Written consents according to Helsinki declaration were taken from all patients and control subjects prior to participation in the study that was approved by the ethical committee of Faculty of Medicine, Benha University.

\section{Consent for publication}

Not applicable.

\section{Competing interests}

The authors declare that they have no competing interests.

Received: 3 May 2020 Accepted: 31 May 2020

Published online: 22 July 2020

\section{References}

1. De Santis M, Di Matteo B, Chisari E, Cincinelli G, Angele P, Lattermann C et al (2018) The Role of Wnt Pathway in the Pathogenesis of OA and Its Potential Therapeutic Implications in the Field of Regenerative Medicine. Hindawi BioMed Res Int 7402947:8

2. Salehi-Abari I (2016) ACR Revised Criteria for Early Diagnosis of primary osteoarthritis. Autoimmune Dis Ther Approach 3(1):113-118

3. Bortoluzzi A, Furini F, Scirè CA (2018) Osteoarthritis and its management Epidemiology, nutritional aspects and environmental factors. Autoimmun Rev 17(11):1097-1104

4. Robinson WH, Lepus CM, Wang Q, Raghu H, Mao R, Lindstrom TM et al (2016) Low-grade inflammation as a key mediator of the pathogenesis of osteoarthritis. Nat Rev Rheumatol 12:580-592

5. Pulsatelli L, Addimanda O, Brusi V, Pavloska B, Meliconi R (2013) New findings in osteoarthritis pathogenesis: therapeutic implicat. Adv Chronic Dis 4(1):23-43 DOl: 10.1177
6. Min S, Wang C, Lu W, Xu Z, Shi D, Chen D et al (2017) Serum levels of the bone turnover markers dickkopf-1, osteoprotegerin, and TNF-a in knee osteoarthritis patients. Clin Rheumatol 36:2351-2358. https://doi.org/10. 1007/s10067-017-3690

7. Goldring SR (2016) Differential mechanisms of de-regulated bone formation in rheumatoid arthritis and spondyloarthritis. Rheumatology:ii56-ii60

8. Morvan F, Boulukos K, Clément-Lacroix P, Roman S, Suc-Royer I, Vayssière B et al (2006) Deletion of a single allele of the DKK-1 gene leads to an increase in bone formation and bone mass. J Bone Miner Res 21:934-945

9. Aletaha D, Neogi T, Silman AJ, Funovits J, Felson DT, Bingham CO 3rd et al (2010) 2010 Rheumatoid Arthritis Classification Criteria: An American College of Rheumatology/European League against Rheumatism Collaborative Initiative. Arthritis Rheum 62:2569-2581.9

10. American College of Rheumatology and WOMAC (2013); American College of Rheumatology and Western Ontario and McMaster Universities Osteoarthritis Index (WOMAC). http://www.rheumatology.Org/practice/ clinical/clinicianresearchers/outcomes-instrumentation/WOMAC.asp.

11. Marty M, Hilliquin P, Rozenberg S, Valat JP, Vignon E, Coste P et al (2009) Validation of the KOFUS (Knee Osteoarthritis Flare-Ups Score). Jnt Bone Spine 76:268

12. Kellgren JH, Lawrence JS (1957) Radiological assessment of osteo-arthrosis. Ann Rheum Dis 16(4):494-502. https://doi.org/10.1136/ard.16.4.494

13. Theologis T, Efstathopoulos N, Nikolaou V, Charikopoulos I, Papapavlos I, Kokkoris P et al (2017) Association between serum and synovial fluid Dickkopf1 levels with radiographic severity in primary knee osteoarthritis patients. Clin Rheumatol 36:1865-1872. https://doi.org/10.1007/s10067-017-3640-7

14. Rao TP, Kühl M (2010) An updated overview on Wnt signaling pathways: a prelude for more. Circ Res 106:1798-1806

15. Zorn AM (2001) Wnt signalling: antagonistic Dickkopfs. CurrBiol 11:R592-R595

16. Niehrs C (2006) Function and biological roles of the Dickkopf family of Wnt modulators. Oncogene 25:7469-7481

17. Wu L, Huang X, Li L, Huang H, Xu R, Luyten W (2012) Insights on biology and pathology of HIF-1/-2, TGF/BMP,Wnt/catenin,and NF-B pathways in osteoarthritis. Curr Pharm Des 18:3293-3312

18. Blom AB, Brockbank SM, van Lent PL, van Beuningen HM, Geurts J, Takahashi N (2009) Involvement of the Wnt signaling pathway in experimental and human osteoarthritis prominent role of Wnt-induced signaling protein 1. Arthritis Rheum 60(2):501-512

19. Clevers H, Nusse R (2012) Wnt/b-catenin signaling and disease. Cell 149(6): 1192-1205

20. Usami Y, Gunawardena AT, Iwamoto M, Enomoto-Iwamoto M (2016) Wnt signaling in cartilage development and diseases: lessons from animal studies. Lab Investig 96(2):186-196

21. Voorzanger-Rousselot N, Ben Tabassi NC, Garnero P (2009) Opposite relationships between circulating Dkk-1 and cartilage breakdown in patients with rheumatoid arthritis and knee osteoarthritis. Ann Rheum Dis 68:1513-1514

22. Honsawek S, Tanavalee A, Yuktanandana P, Ngarmukos S, Saetan N, Tantavisut S (2010) Dickkopf-1 (Dkk-1) in plasma and synovial fluid is inversely correlated with radiographic severity of knee osteoarthritis patients. BMC Musculoskelet Disord 11:257

23. Hassan WA, Behiry EG (2018) Decreased synovial levels of dickkopf-1 are associated with radiological progression in knee osteoarthritis patients. Rheumatol Rehabil. https://doi.org/10.1136/annrheumdis-eular.4608

24. Seror R, Boudaoud S, Pavy S, Nocturne G, Schaeverbeke T, Saraux A et al (2016); Increased Dickkopf-1 in Recent-onset Rheumatoid Arthritis is a New Biomarker of Structural Severity. Data from the ESPOIR Cohort Article number: 18421.

25. Diarra D, Stolina M, Polzer K, Zwerina J, Ominsky MS, Dwyer D et al (2007) Dickkopf-1 is a master regulator of joint remodeling. Nat Med 13:156-163

26. Lane NE, Nevitt MC, Lui LY, de Leon P, Corr M (2007) Wnt signaling antagonists are potential prognostic biomarkers for the progression of radiographic hip osteoarthritis in elderly Caucasian women. Arthritis Rheum 56(10):3319-3325

27. Leijten JC, Emons J, Sticht C, van Gool S, Decker E, Uitterlinden A et al (2012) Gremlin 1, Frizzled-related protein, and Dkk-1 are key regulators of human articular cartilage homeostasis. Arthritis Rheum 64:3302-3312

\section{Publisher's Note}

Springer Nature remains neutral with regard to jurisdictional claims in published maps and institutional affiliations. 